\title{
Bioinspired tumor-homing nanosystem for precise cancer therapy via reprogramming of tumor-associated macrophages
}

\author{
Yanyu Huang ${ }^{1}$, Chaoming Mei', Yiqiao Tian', Tianqi Nie', Zhuang Liu $\mathbb{1}^{2}$ and Tianfeng Chen (iD)
}

\begin{abstract}
Rational design of smart nanosystems with high biological safety is a critical milestone for realizing precise imagingguided cancer theranostics. Herein, a bioinspired nanosystem was designed by camouflaging SPIO@DOX-ICG nanoparticles with cancer cell membrane (CCM) to realize precise cancer treatment through simultaneous chemotherapy, hyperthermia-therapy, and radiotherapy. CCM surface decoration preserves the cancer adhesion molecules and surface antigens in the nanosystem, endowing the nanosystem with tumor-homing ability and high biocompatibility. Guided by dual-modal imaging, the nanosystem specifically accumulated in the tumor region and achieved synergistic anticancer effects after combined treatment, without causing toxic side effects in major organs. Interestingly, the combined treatment also antagonized tumor hypoxia and reprogrammed the polarization of tumor associated macrophages to the antitumor M1 phenotype. Taken together, this study offers a smart strategy for designing a bioinspired tumor-homing nanosystem for precise cancer therapy.
\end{abstract}

\section{Introduction}

Conventional cancer treatment methods, including surgery, chemotherapy, radiotherapy, photothermal therapy, and immunotherapy, often lead to treatment failure due to tumor complexity, diversity, and heterogeneity ${ }^{1}$. Multimodal diagnostic and therapeutic agents (theranostics) based on cooperative enhancement between two or more treatments are expected to achieve synergistic therapeutic effects and real-time monitoring of treatment efficacy $^{2-4}$. Clinically, comprehensive imaging and therapeutic strategies have been shown to enhance the therapeutic effects for cancer patients ${ }^{5,6}$. The combination of chemotherapy with hyperthermia and/or radiotherapy (CHRT) was demonstrated to have an enhanced anticancer effect and long-term survival benefits ${ }^{7}$. However,

\footnotetext{
Correspondence: Zhuang Liu (zliu@suda.edu.cn) or

Tianfeng Chen (tchentf@jnu.edu.cn)

'The First Affiliated Hospital, and Department of Chemistry, Jinan University, 510632 Guangzhou, China

${ }^{2}$ Institute of Functional Nano and Soft Materials (FUNSOM), Jiangsu Key Laboratory for Carbon-Based Functional Materials and Devices, Soochow University, Suzhou 215000, China
}

conventional chemotherapeutic drugs (e.g., doxorubicin) and imaging agents (e.g., indocyanine green) showed poor pharmacokinetic characteristics and tumor selectivity and thus caused significant side effects ${ }^{8,9}$. Therefore, it is necessary and significant to integrate diversified therapeutic and imaging agents into one formulation to realize the goal of (i) multimodal theranostic modality with tumor selectivity and (ii) synergistic imaging/therapeutic outcomes under lower drug dosages.

In recent decades, numerous nanomedicines have been developed to improve anticancer efficacy through the enhanced permeability and retention (EPR) effect ${ }^{10}$ and cancer-targeted design ${ }^{11,12}$. However, most exogenous nanoparticles constructed by exploiting organic or inorganic materials cause innate immunogenicity, are rapidly cleared by the reticuloendothelial system (RES) system, and exhibit poor biodegradability and long-term toxicity ${ }^{4}$. Furthermore, the off-target effect of chemically modified ligands in vivo minimizes the tumor selectivity of nanoparticles ${ }^{13}$. Previous studies have also found that nanoparticles in the tumor tissues are easily internalized by 
tumor-associated macrophages (TAMs) that are recruited in the tumor microenvironment ${ }^{14,15}$. This effect has been utilized for immune-modulating cancer therapies with diagnostic nanoparticles ${ }^{3,4,6}$, or to shuttle the nanoparticle-conjugated therapeutic drugs to tumors ${ }^{7,8,15}$. Therefore, reprogramming TAMs from the pro-tumoral M2 phenotype towards the antitumor M1 phenotype would be an effective strategy to induce tumor regression.

Fabrication of bioinspired nanosystems by exploiting naturally sourced-nanomaterials promises a new horizon for next-generation nanomedicine ${ }^{16}$. Specifically, the utilization of cell membranes as biomimetic nanocarriers have attracted considerable attention due to their ability to maintain cell surface antigens, such as cell adhesion molecules $(\mathrm{CAMs})^{17}$, and their outstanding biocompatibility $^{18,19}$. Interestingly, cancer cell membrane (CCM)-biomimetic nanoparticles, which are equipped with special membrane antigens, have been confirmed to show higher imaging and therapeutic properties, specific recognition, and long blood circulation by mimicking the migration and invasion of tumor cells $\mathrm{s}^{20-22}$. Therefore, CCM-camouflaged nanoparticles can effectively navigate to tumors by mimicking the metastatic and invasive properties of tumor cells in vivo.

Therefore, we have designed a bioinspired nanosystem by camouflaging SPIO@DOX-ICG nanoparticles with cancer cell membrane (CCM) to realize precise cancer treatment via simultaneous chemotherapy, hyperthermia-therapy, and radiotherapy. The CCM surface decoration preserves cancer adhesion molecules, such as adherent molecules and surface antigens, in the nanosystem, which endows the nanosystem with tumorhoming ability and high biocompatibility. As guided by dual-modal imaging, the nanosystem specifically accumulated in the tumor region and achieved synergistic anticancer effects after combined treatment, without causing toxic side effects in major organs. Interestingly, the bioinspired nanosystem also antagonized tumor hypoxia and reprogrammed the polarization of tumor associated macrophages to the anti-tumor M1 phenotype. Taken together, this study offers a smart design strategy to create a bioinspired tumor-homing nanosystem for precise cancer therapy.

\section{Materials and methods Materials}

Poly-L-lysine (1\%) was bought from Sigma-Aldrich Company (St. Louis, MO USA). The amino groupmodified superparamagnetic iron oxide (SPIO- $\mathrm{NH}_{2}$ ) was bought from Nanjing Xianfeng Nanotechnology Company (Nanjing, China). Indocyanine (ICG) was bought from Aladdin Company (Shanghai, China). A bicinchoninic acid (BCA) kit was purchased from Thermo Fisher Scientific Inc. (Waltham, U.S.) Dulbecco's modified
Eagle's medium (DMEM) and fetal bovine serum (FBS) were purchased from Gibco $^{\circledR}$ Thermo Fisher Scientific Inc. (Waltham, U.S.) Antibodies targeting MUC-1 (\#4538S), Galectin-3 (\#97985S) and CD47 (SAB1401057) were bought from Cell Signaling Technology, Inc. (Boston, U.S.) and Sigma-Aldrich Company (St. Louis, MO USA), respectively. The mice used in this study were treated with care in compliance with the guidelines for the Care and Use of Laboratory Animals. All animal experiments were conducted with the approval of the Animal Experimentation Ethics Committee of Jinan University.

\section{Fabrication of CCM/IDINPs}

Briefly, $1.3 \mathrm{~mL}$ of doxorubicin (DOX, $9.22 \mathrm{mM}$ ) was added to $30 \mathrm{~mL}$ poly-lysine solution (1\%), followed by the addition of $2.48 \mathrm{~mL}$ of DTSSP $(2.5 \mathrm{mg} / \mathrm{mL})$ and $10 \mathrm{~mL}$ of amine-modified SPIO $(1 \mathrm{mg} / \mathrm{mL})$. The $\mathrm{pH}$ was adjusted to 9.0 by the addition of $0.1 \mathrm{M} \mathrm{KOH}$. After continuous stirring for $24 \mathrm{~h}$, the mixed solution was added to $400 \mu \mathrm{L}$ of ICG $(80 \mathrm{mg} / \mathrm{mL})$ and stirred for another $24 \mathrm{~h}$. Afterwards, the final mixture was dialyzed against diluted water for $24 \mathrm{~h}$ to remove excess DTSSP, DOX, and ICG. The asprepared nanosystem was marked as IDINPs. The molecular weight of cut-off used for dialysis was $3500 \mathrm{kDa}$.

\section{Preparation of CCM}

The CCM was obtained by procedures described in previous studies ${ }^{17}$. First, K562 cells were centrifuged at $1500 \mathrm{rpm}$ for $10 \mathrm{~min}$. After that, the cells were suspended in a hypotonic lysing buffer and homogenized in a homogenizer for 20 passes. The cell lysate was spun down at $3200 \times g$ for $10 \mathrm{~min}$. The supernatant was collected, and the pellet was rehomogenized following the same procedure 5 times. The collected supernatant was centrifuged at $20,000 \times g$ for $20 \mathrm{~min}$. After that, the pellet was resuspended in the hypotonic lysing buffer before centrifugation at $100,000 \times g$ for $20 \mathrm{~min}$. The pellet was washed with $10 \mathrm{mM}$ Tris- $\mathrm{HCl}$ and $1 \mathrm{mM}$ Ethylenediaminetetraacetic acid (EDTA) and centrifuged at 100,000 $\times g$ for $20 \mathrm{~min}$. The pellet was collected as purified CCM.

\section{Preparation of CCM/IDINPs}

The nanoscale CCM vesicles were formed by sequential physical extrusion through $800 \mathrm{~nm}$ and $400 \mathrm{~nm}$ porous polycarbonate membranes. Afterwards, the CCM vesicles were mixed with the as-prepared IDINPs with sonication at $37 \mathrm{kHz}$ for $30 \mathrm{~min}$, followed by sequential physical extrusion through a $200 \mathrm{~nm}$ and a $100 \mathrm{~nm}$ polycarbonate membrane. The final product, CCM-coated IDINPs (CCM/IDINPs), was used for subsequent experiments. The DOX concentration in CCM/IDINPs was quantified by measuring the fluorescence intensity of DOX with the excitation and emission wavelength set at $480 \mathrm{~nm}$ and $590 \mathrm{~nm}$, respectively. The ICG concentration in CCM/IDINPs was quantified by 
measuring the absorbance of ICG with the wavelength set at $805 \mathrm{~nm}$

\section{Chemical characterization of CCM/IDINPs}

The morphology of CCM/IDINPs was observed using transmission electron microscopy (TEM, JEM-2100F, JEOL, Japan). The elemental composition of CCM/ IDINPs was characterized by dark-field scanning TEM (DF-STEM, JEOL, Japan) and energy-dispersive X-ray spectrometry (EDS, JEOL, Japan). The UV-vis- NIR spectra were detected on a UV-vis-NIR spectrophotometer with the wavelength ranging from $400 \mathrm{~nm}$ to $880 \mathrm{~nm}$. The zeta potential of the nanosystem was examined with a Nano-ZS instrument (Malvern Instruments Limited, England).

\section{Photothermal effects of CCM/IDINPs}

First, the CCM/IDINPs and ICG with an equivalent concentration of $128 \mathrm{mg} / \mathrm{L}$ were exposed to irradiation with an NIR laser (Laser Technol. Co., Ltd of Xi'an, China) for $1200 \mathrm{~s}$, with the laser density set at $0.5,0.75$, and $1.0 \mathrm{~W} / \mathrm{cm}^{2}$. Second, the CCM/IDINPs and ICG were exposed to a switch-on and switch-off transition of the NIR laser for 10 cycles. In each cycle, the ICG or CCM/IDINP sample was irradiated with an 808-nm laser $\left(1.0 \mathrm{~W} / \mathrm{cm}^{2}\right)$ for $150 \mathrm{~s}$. Subsequently, the laser was shut off for $150 \mathrm{~s}$. The temperature of ICG or CCM/ IDINPs was recorded every $30 \mathrm{~s}$ with an infrared thermograph (Fluke, U.S.).

\section{Magnetic properties of CCM/IDINPs}

The magnetic properties of SPIO, IDINP, and CCM/ IDINP solutions with an equivalent $\mathrm{Fe}$ concentration ranging from $25-400 \mu \mathrm{M}$ were determined by using a $1.5 \mathrm{~T}$ Signa HDxt superconductor clinical Magnetic resonance (MR) system (GE Medical, Milwaukee, U.S.). The relaxivity value $\left(\mathrm{r}_{2}\right)$ of free SPIO, IDINPs and CCM/ IDINPs, which is defined as $1 / T_{2}$, was measured with the following parameters: field of view, $120 \times 120 \mathrm{~mm}^{2}$; TR, $1860 \mathrm{~ms}$, TE, $85.1 \mathrm{~ms}$; slice thickness, $2.0 \mathrm{~mm}$; number of slices, 9; thickness, $2 \mathrm{~mm}$.

\section{Cell culture}

The cell lines used in this study, including human MG63 osteosarcoma cells, human K562 myelogenous leukemia line cells, human nonsmall cell lung cancer A549 cells, human SW480 colorectal adenocarcinoma cells, human A375 melanoma cells, human HepG2 hepatocellular carcinoma cells, human MCF-7 breast carcinoma cells, human MGC-803 gastric carcinoma cells, human L02 hepatic cells, and murine RAW264.7 macrophage cells, were purchased from American Type Culture Collection (ATCC, Manassas, Virginia). All the cell lines were incubated in DMEM with penicillin (100 units $/ \mathrm{mL}$ ), streptomycin (50 units/mL), and fetal bovine serum (10\%) at $37^{\circ} \mathrm{C}$ in a $\mathrm{CO}_{2}$ incubator.

\section{Expression of MUC-1 and Galectin-3 on different cells}

The expression of MUC-1 and Galectin-3 on red blood cells (RBCs), MG-63 cells, K562 cells, A549 cells, SW480 cells, A375 cells, HepG2 cells, MCF-7 cells, MGC-803 cells, L02 cells, K562 lysate, K562 membranes, IDINPs, and CCM/IDINPs was determined by Western blot analysis as we previously described ${ }^{23}$. $\beta$-Actin was used as the loading control. The expression of the examined proteins was quantified using ImageJ Software (National Institutes of Health, U.S.).

\section{Cytotoxicities of CCM/IDINPs and laser and X-ray irradiation in combination}

Briefly, cells were seeded in 96-well plates at a density of $2 \times 10^{4}$ cells $/ \mathrm{mL}$. After cell attachment, MG-63 cells were treated with DOX, ICG, IDINPs and CCM/ IDINPs for $72 \mathrm{~h}$ at equivalent concentrations of $1 \mu \mathrm{M}$ DOX and $15.4 \mathrm{mg} / \mathrm{L}$ ICG. After $12 \mathrm{~h}$ of drug administration, MG-63 cells were irradiated with an NIR laser (808 $\mathrm{nm}, 1.0 \mathrm{~W} / \mathrm{cm}^{2}$ ) for $3 \mathrm{~min}$ and X-ray irradiation (2 Gy). After $72 \mathrm{~h}$ of drug incubation, the cytotoxicities of the combined treatment with CCM/IDINPs, the NIR laser and X-rays in MG-63 cells were measured with an MTT assay ${ }^{23}$.

\section{Efficacy of cellular uptake of CCM/IDINPs}

MG-63 cells and RAW264.7 cells were incubated with SPIO, IDINPs and CCM/IDINPs with an equivalent concentration of Fe $(200 \mu \mathrm{M})$ for $8 \mathrm{~h}$. The efficacy of SPIO, IDINP, and CCM/IDINP uptake was quantified by measuring the $\mathrm{T}_{2}$-weighted signal of SPIO in a $1.5 \mathrm{~T} \mathrm{MR}$ scanner.

\section{Flow cytometric analysis}

The cell cycle of MG-63 cells after treatment with $2 \mu \mathrm{M}$ CCM/ININPs combined with the 808 -nm laser $\left(1.0 \mathrm{~W} / \mathrm{cm}^{2}\right)$ and X-ray (2 Gy) irradiation for $48 \mathrm{~h}$ was examined by flow cytometric analysis as previously described ${ }^{23}$.

\section{Measurement of intracellular radical oxygen species (ROS) generation}

ROS generation in MG-63 cells after the combined CCM/ININP, $808 \mathrm{~nm}$ laser $\left(1.0 \mathrm{~W} / \mathrm{cm}^{2}\right)$ and X-ray (2 Gy) treatments was determined by using DCFH-DA as a fluorescent probe ${ }^{24}$.

\section{Drug release behavior of CCM/IDINPs in vitro}

Five duplicate CCM/IDINP samples were suspended separately in $10 \mathrm{~mL}$ of GSH $(20 \mu \mathrm{M})$ solution at $\mathrm{pH} 7.4$, GSH $(20 \mu \mathrm{M})$ at $\mathrm{pH} 6.8$, GSH $(10 \mathrm{mM})$ at $\mathrm{pH} 7.4$, $\mathrm{GSH}(10 \mathrm{mM})$ at $\mathrm{pH} 7.4$ after irradiation with a laser 
$\left(1.0 \mathrm{~W} / \mathrm{cm}^{2}\right)$, GSH $(10 \mathrm{mM})$ at $\mathrm{pH} 7.4$ after irradiation with a laser $\left(1.0 \mathrm{~W} / \mathrm{cm}^{2}\right)$ and $\mathrm{X}$-rays $(2 \mathrm{~Gy})$ with constant shaking in dark tubes at $37^{\circ} \mathrm{C}$. At specific intervals, the release rate of DOX was measured as we previously described ${ }^{23}$. The morphology of CCM/IDINPs after the incubation with different treatments was observed via TEM.

\section{Intracellular trafficking of CCM/IDINPs in MG-63 cells}

The intracellular trafficking of CCM/IDINPs in MG-63 cells was observed by costaining of the cytoskeleton and nucleus. Briefly, the cytoskeleton was stained with Fluor 488 phalloidin (green) and Hoechst 33342 (blue). MG-63 cells were seeded at a density of $5 \times 10^{4}$ cells $/ \mathrm{mL}$ for $24 \mathrm{~h}$. After attachment, the MG-63 cells were incubated with an $8 \mu \mathrm{M}$ solution of CCM/IDINPs for $12 \mathrm{~h}$. At different time points, the red fluorescence of DOX from the CCM/ IDINPs was observed under a fluorescence microscope (EVOS ${ }^{\oplus}$ FL Auto Imaging System, AMAFD1000, U.S.).

\section{Stability of CCM/IDINPs in a physiological environment}

To evaluate the stability of CCM/IDINPs in a physiological environment, the size of CCM/IDINPs in PBS, DMEM (containing 10\% FBS) and human plasma was monitored for $120 \mathrm{~h}$ using a Nano-ZS instrument (Malvern Instruments Limited, England).

\section{Pharmacokinetics of CCM/IDINPs in vivo}

Twelve male SD rats (7 weeks old) were randomly divided into 4 groups ( $n=3$ per group). DOX, ICG, IDINPs and CCM/IDINPs were injected intravenously at an equivalent dosage of $80 \mu \mathrm{g}$ DOX and $2.1 \mathrm{mg}$ ICG per $\mathrm{kg}$ (injection volume: $0.8 \mathrm{~mL}$ ). At 1, 2, 4, 6, 10, 24, 36, 56, and $80 \mathrm{~h}$, blood was gathered from the retro-orbital plexus of the rat eyes and immediately spun down at $3000 \mathrm{rpm}$. The plasma from the supernatant was collected. DOX and ICG were extracted from the plasma by dissolving the plasma in acidic isopropanol (containing $0.75 \mathrm{M} \mathrm{HCl}$ solution) at $-20^{\circ} \mathrm{C}$ for $8 \mathrm{~h}$. Subsequently, the samples were spun down at $17,709 \times \mathrm{g}$ for $20 \mathrm{~min}$, and the supernatant was collected. The DOX concentration in the supernatant was measured with the excitation and emission wavelengths set at $480 \mathrm{~nm}$ and $590 \mathrm{~nm}$. The concentration of ICG in the supernatant was measured with the wavelength set at $805 \mathrm{~nm}$. The pharmacokinetic parameters were analyzed using Winonlin 3.3 software (Pharsight, U.S.).

\section{MRI and NIRFI of CCM/IDINPs in vivo}

Real-time imaging in vivo was performed to identify the biodistribution of CCM/IDINPs in MG-63 xenograftbearing nude mice. First, the biodistribution of CCM/ IDINPs in the MG-63 xenograft-bearing nude mice was examined with a $1.5 \mathrm{~T}$ MR scanner. The nude mice were randomly divided into 3 groups ( $n=3$ per group). Equivalent doses of SPIO, IDINPs and CCM/IDINPs at concentrations of $0.5 \mathrm{mg} / \mathrm{kg}$ (SPIO concentration, injection volume: $0.2 \mathrm{~mL}$ ) were intravenously injected into the mice via the caudal vein. Dynamic MRI was performed by collecting the $\mathrm{T}_{2}$-weighted signal of SPIO in the mice. The accumulative concentration of SPIO was quantified as the percentage difference of transverse relativity $\left(\triangle R_{2}{ }^{*}\right)$.

For NIRFI in vivo, equivalent doses of ICG, IDINPs and CCM/IDINPs at concentrations of $26.5 \mathrm{mg} / \mathrm{kg}$ (ICG concentration, injection volume: $0.2 \mathrm{~mL}$ ) were intravenously injected. Dynamic fluorescence imaging was performed by collecting the NIR signal of ICG in the mice using a live imaging system (NEO image FOBI, Neoscience, Korea), with the observation time set at 12 $\mathrm{h}, 24 \mathrm{~h}, 36 \mathrm{~h}, 48 \mathrm{~h}$, and $72 \mathrm{~h}$ postinjection. At the end of the experiment, the mice were sacrificed, and the brain, heart, liver, spleen, lungs, kidneys and tumor were subjected to ex vivo imaging. The accumulative amount of ICG and CCM/IDINPs in each organ and the tumor was quantified by measuring the concentration of ICG at $805 \mathrm{~nm}$.

\section{Hemolysis rate of CCM/IDINPs}

To assess the biocompatibility of CCM/IDINPs in blood, the hemolysis rate of CCM/IDINPs was measured. Briefly, $660.8 \mu \mathrm{g} / \mathrm{mL}$ SPIO, $1.3 \mathrm{mg} / \mathrm{mL}$ ICG, $47.2 \mu \mathrm{g} / \mathrm{mL}$ DOX, $47.2 \mu \mathrm{g} / \mathrm{mL}$ IDINPs (containing $660.8 \mu \mathrm{g} / \mathrm{mL}$ SPIO, $47.2 \mu \mathrm{g} / \mathrm{mL}$ DOX and $1.3 \mathrm{mg} / \mathrm{mL}$ ICG), and $47.2 \mu \mathrm{g} / \mathrm{mL}$ CCM/IDINPs (containing $660.8 \mu \mathrm{g} / \mathrm{mL}$ SPIO, $47.2 \mu \mathrm{g} / \mathrm{mL}$ DOX and $1.3 \mathrm{mg} / \mathrm{mL}$ ICG) were incubated with human RBCs for $6 \mathrm{~h}$. RBCs treated with Triton-X $(10 \mathrm{~g} / \mathrm{L})$ were used as the positive control. RBCs treated with PBS were used as the negative control. After that, the RBCs were spun down, and the absorbance of the supernatant was detected at $540 \mathrm{~nm}$. The hemolysis rate was calculated based on the following formula:

$$
\text { Hemolysis rate }(\%)=\frac{A_{\text {Sample }}-A_{\text {Negtive control }}}{A_{\text {Positiv control }}-A_{\text {Negative control }}} \times 100 \%
$$

The morphology of RBCs after incubation with the different treatments was captured under a microscope (DS-Fi2, magnification: $\times 40$, Nikon, Japan).

\section{Administration mode and anticancer ability of CCM/IDINPs in vivo}

The osteosarcoma xenograft model was constructed by injecting $2 \times 10^{6}$ MG-63 cells into the armpits near the left fore of $\mathrm{BALB} / \mathrm{c}$ nude mice. When the tumor volume reached $150 \mathrm{~mm}^{3}$ after inoculation, the tumorbearing mice were used for the animal study. The osteosarcoma xenograft nude mice were randomly divided into 13 groups ( $n=6$ per group). Equivalent concentrations of ICG $(26.5 \mathrm{mg} / \mathrm{kg})$, SPIO $(14 \mathrm{mg} / \mathrm{kg})$, 
DOX (1 mg/kg), SPIO@DOX (containing $1 \mathrm{mg} / \mathrm{kg}$ DOX and $14 \mathrm{mg} / \mathrm{kg}$ SPIO), IDINPs (containing $1 \mathrm{mg} / \mathrm{kg}$ DOX, $14 \mathrm{mg} / \mathrm{kg}$ SPIO and $26.5 \mathrm{mg} / \mathrm{kg}$ ICG), and CCM/ININPs (containing $1 \mathrm{mg} / \mathrm{kg}$ DOX, $14 \mathrm{mg} / \mathrm{kg}$ SPIO and $26.5 \mathrm{mg} /$ $\mathrm{kg}$ ICG) were intravenously injected into the mice every 2 days (injection volume: $200 \mu \mathrm{L}$ ). After $12 \mathrm{~h}$ of drug administration, the tumor region of each mouse was irradiated with an NIR laser $\left(808 \mathrm{~nm}, 1.0 \mathrm{~W} / \mathrm{cm}^{2}\right)$ for $5 \mathrm{~min}$ and with $\mathrm{X}$-rays (2 Gy). The control group received an equal volume of saline. After 12 days of drug administration, the temperature in tumor region was recorded with an infrared thermograph. The tumor volume and body weight of each mouse was determined every 2 days. The tumor volume was calculated using the following equation: Volume $\left(\mathrm{mm}^{3}\right)=1 \times \mathrm{w}^{2} / 2$, with 1 being the maximal length and $\mathrm{w}$ being the maximal width. After 19 days of drug administration, the mice were sacrificed, and the blood was gathered for blood biochemical analysis. The heart, liver, spleen, lung, kidney and tumor were obtained and weighed. The accumulative concentration of ICG, DOX, IDINPs, and CCM/IDINPs in each organ and tumor region was quantified as described in the section "MRI and NIRFI imaging of CCM/IDINPs in vivo".

\section{MRI acquisition}

$\mathrm{T}_{2}$-weighted MRI of the nude mice with subcutaneous osteosarcoma xenografts was conducted 19 days after the different treatments with a $1.5 \mathrm{~T}$ MR scanner (GE Medical, Milwaukee, U.S.) equipped with a mouse body volume coil. The indices of standard ADC and slow ADC were employed to evaluate the necrotic degree of the tumors in vivo.

\section{H\&E staining and immunohistochemistry (IHC) analysis}

The tissues, including the heart, liver, spleen, lung, kidney and tumor, were fixed, embedded, sectioned and stained with hematoxylin and eosin for histological evaluation under an optical microscope (magnification: $\times 20$, Nikon, Japan). The expression of TNF- $\alpha$, Hsp70, and HIF1- $\alpha$ was determined via IHC as we previously described ${ }^{23}$.

\section{Flow cytometric analysis of the ratio of M2/M1 macrophages}

Tumors were mechanically disrupted and incubated in $2 \mathrm{mg} / \mathrm{mL}$ collagenase type IV diluted in DMEM for $0.5 \mathrm{~h}$ at $37^{\circ} \mathrm{C}$. Collagenase activity was stopped by adding an equal volume of DMEM supplemented with $10 \%$ fetal bovine serum (FBS) followed by centrifugation at $300 \times g$ for $10 \mathrm{~min}$. Cell pellets were filtered through a $100-\mu \mathrm{m}$ nylon mesh cell strainer. Cultured cells were collected following the standard procedure. The macrophages from tumor cells were stained with FITC-labeled F4/80 (dilution 1:100, Abcam, Cambridge, England). Alexa
Fluor $^{\circledR}$ 647-labeled CD206 antibody (dilution 1:100, Abcam, Cambridge, England) and PE-labeled iNOS antibody (dilution 1:100, Abcam, Cambridge, England) were employed to label M2 and M1 macrophages. Isotype controls were employed to establish background fluorescence. The data were acquired on a Beckman Coulter Gallios flow cytometer (Beckman Coulter, Miami, U.S.) and analyzed using FlowJo software (Ver. 7.6.1, Treestar U.S.).

\section{Statistical analysis}

All experiments were carried out in triplicate, and the results are expressed as the mean \pm SD. Statistical analysis was performed using SPSS statistical software, version 13 (SPSS Inc., Chicago, IL). Differences between two groups were analyzed using a two-tailed Student's $t$-test. Differences with $P<0.05\left(^{*}\right)$ or $P<0.01\left(^{* * *}\right)$ were considered statistically significant. Differences among multiple groups were analyzed via one-way analysis of variance with Tukey's post hoc test.

\section{Results and discussion}

\section{Rational design of the CCM-camouflaged nanosystem}

The smart CCM-camouflaged nanosystem (CCM/ IDINPs) was designed and synthesized via sequential assembly of SPIO@DOX-ICG, extrusion and coating of CCM on the nanoparticles. As illustrated in Fig. 1a, SPIO was linked to DOX by GSH-sensitive 3,3'-dithiobis (sulfosuccinimidyl propionate) (DTSSP), followed by the $\pi-\pi$ stacking of ICG and DOX, yielding SPIO@DOX-ICG (IDINPs). To fabricate the CCM-based nanosystem, leukemic cell-oriented membrane vesicles were prepared according to the procedure of stepwise physical extru$\operatorname{sion}^{17}$. The obtained CCM was mixed with the asprepared IDINPs under sonication, and the components were extruded together through a $100-\mathrm{nm}$ polycarbonate membrane, yielding CCM-coated IDINPs (CCM/IDINPs). The loading efficacy of DOX, ICG, and SPIO in CCM/ IDINPs was $1.8 \%, 47.2 \%$, and $30.0 \%$, respectively.

Transmission electronic microscopy (TEM) images showed that the morphology of aminated SPIO, SPIO@DOX, and IDINPs was spherical, with an average size of $7.9 \mathrm{~nm}, 10.8 \mathrm{~nm}$, and $12.5 \mathrm{~nm}$, respectively. In addition, the lattice fringe on SPIO $(0.14 \mathrm{~nm})$ disappeared on the SPIO@DOX and IDINPs, which was ascribed to the interaction between DOX and ICG on the SPIO surface. The coating of the pie-like CCM on IDINPs enlarged the size of the nanosystem to $96.9 \mathrm{~nm}$ (Fig. 1b) and shifted the zeta potential to $-15.0 \mathrm{mV}$ (Fig. S1a). The results of an elemental composition study (Fig. 1c) showed that the elements $\mathrm{P}, \mathrm{S}$, and $\mathrm{Ca}$ in $\mathrm{CCM}$ were well dispersed around the Fe atoms, which further demonstrated the coating of CCM on IDINPs. UV-vis spectra (Fig. S1b) demonstrated the presence of DOX and ICG in CCM/IDINPs, as 

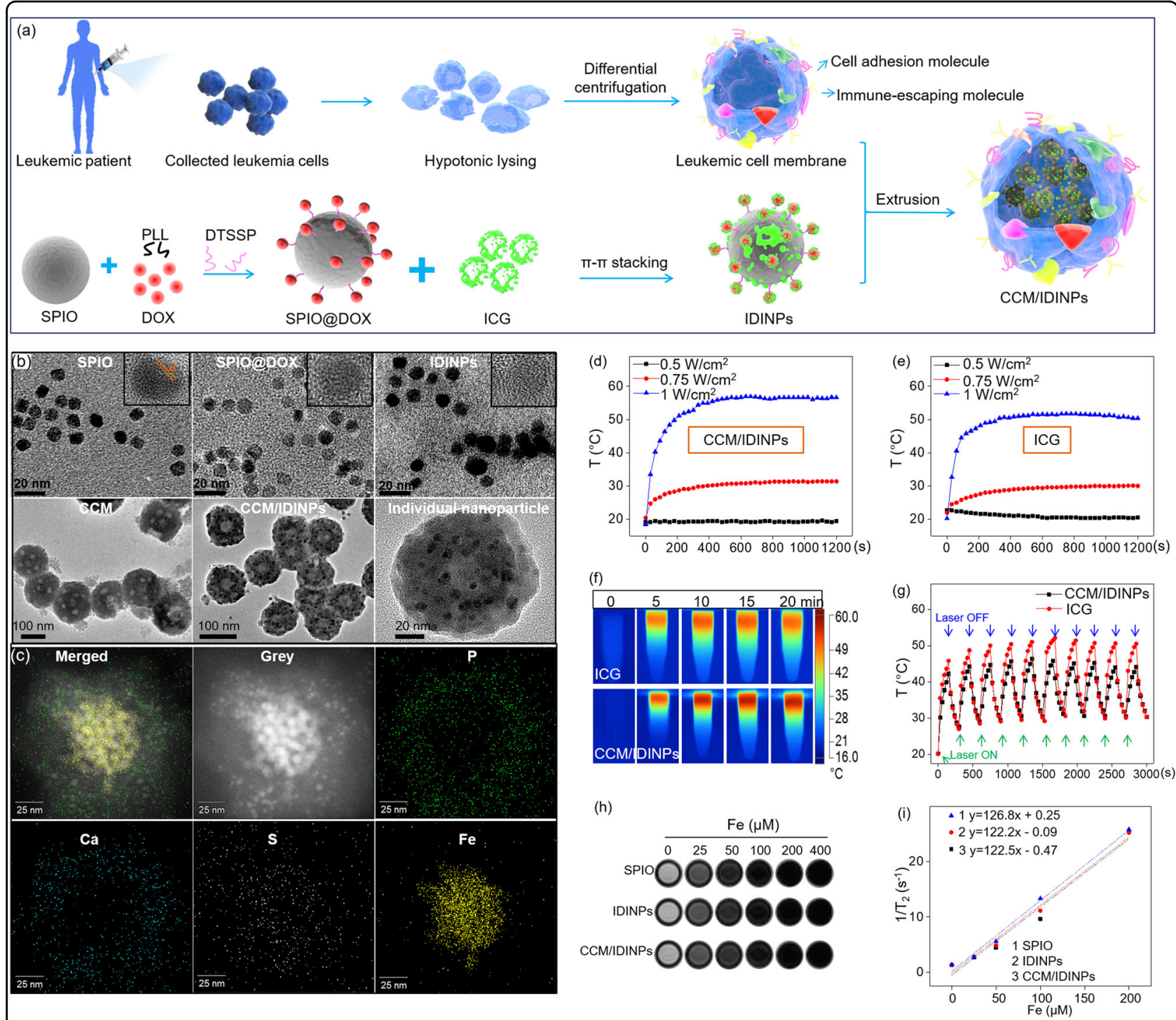

Fig. 1 Fabrication, photothermal effect, and magnetic property of CCM/IDINPs. a Scheme illustrating the preparation process for CCM/IDINPS. TEM images of (b) SPIO, SPIO@DOX, IDINPs, CCM, CCM/IDINPs and individual nanoparticle of the CCM/IDINP system; scale bar $=20 \mathrm{~nm}$ and $100 \mathrm{~nm}$. c DF-TEM images of CCM/IDINPs, scale bar $=25 \mathrm{~nm}$. Temperature curves of CCM/IDINPs (d) and ICG (e) after NIR laser irradiation with the laser density set at $0.5,0.75$ and $1.0 \mathrm{~W} / \mathrm{cm}^{2}$ for $1200 \mathrm{~s}$. f Representative images of CCM/IDINPs and ICG with continuous irradiation with an NIR laser at different time points $\left(1.0 \mathrm{~W} / \mathrm{cm}^{2}\right)$. g Temperature changes of CCM/IDINPs and ICG solution with NIR laser $\left(1.0 \mathrm{~W} / \mathrm{cm}^{2}\right)$ switch-on and switch-off irradiation for 10 cycles. $\mathbf{h}$ T$_{2}$-weighted MR images of CCM/IDINPs, IDINPs, and SPIO with different Fe concentrations ranging from 25 to $400 \mu \mathrm{M}$. i Fitted curves of the $T_{2}$-weighted relaxation rate of CCM/IDINPs, IDINPs, and SPIO versus Fe concentration

evidenced by their characteristic absorbance at $480 \mathrm{~nm}$ and $805 \mathrm{~nm}$, respectively. The results of Fourier transform infrared (FT-IR) spectroscopy (Equinox 55, Bruker, Billerica, MA, USA) further demonstrated the presence of SPIO, DOX, ICG, and CCM in the CCM/IDINP nanosystem (Fig. S2).

\section{Enhanced photothermal ability of CCM/IDINPs with intense MR signals}

The photothermal ability of CCM/IDINPs was examined. Figure $1 \mathrm{~d}-\mathrm{f}$ shows that CCM/IDINPs overwhelmed
IDINPs and ICG by rapidly elevating the temperature upon irradiation with an 808-nm laser at different laser densities. Moreover, the packaging of ICG in CCM/ IDINPs improved the photostability of ICG, as evidenced by the reducible photothermal effect with 10 laser switchon/switch-off cycles (Fig. 1g).

SPIO is used as a $\mathrm{T}_{2}$-weighted contrast agent for clinical $\mathrm{MRI}^{25}$. In this study, measurement of the $\mathrm{T}_{2}$ weighted signal of SPIO with a $1.5 \mathrm{~T}$ MR scanner showed an intense $\mathrm{T}_{2}$-weighted signal of CCM/IDINPs compared with SPIO and IDINPs (Fig. 1h). In addition, 
the relaxivity of CCM/IDINPs, which characterizes the sensitivity of the MR signal, was similar to that of SPIO and IDINPs, suggesting that packaging of SPIO into the nanosystem minimally weakened the intensity of the $\mathrm{T}_{2}$-weighted signal (Fig. 1i).

\section{In vitro anticancer ability is enhanced by CCM/IDINPs and} HRT in combination

First, to identify the most vulnerable cancer cell line as a cell model for in vitro study, the expression of CAMs, including Galectin-3 and MUC-1, on different cancer cells (e.g., MG-63, A549, SW380, A375, HepG2, MCF-7, and MGC-803 cells) and normal liver L02 cells was measured by Western blotting. Our results showed that MG-63 cells are the most sensitive cells for intercellular adhesion, as demonstrated by the highest expression of MUC-1 and Galectin-3 on MG-63 cells compared with other cancer cells (Fig. S3). Therefore, MG-63 cells were selected as the cell model for the following experiments. Despite that, we determined that $\mathrm{K} 562$ cells expressed a higher level of MUC-1 and Galectin-3 than MG-63 cells (Fig. 2a). Therefore, the K562 cell membrane was coated on the surface of IDINPs. The expression of Galectin-3 and MUC-1 on CCM/IDINPs was confirmed by Western blotting (Fig. 2b).

To investigate the cytotoxicity of CCM/IDINPs and HRT in combination, the cytotoxicity of different treatments was measured with MTT assays (Fig. 2c). We found that CCM-camouflaged IDINPs provoked the anticancer ability of free DOX and IDINPs against MG-63 cells at a significant level. After irradiation with an NIR laser $\left(808 \mathrm{~nm}, 1.0 \mathrm{~W} / \mathrm{cm}^{2}\right)$, the temperature of CCM/IDINPs increased to $48.8^{\circ} \mathrm{C}$ in MG-63 cells within $3 \mathrm{~min}$ (Fig. 2d) and subsequently inhibited the cell viability to $35.5 \%$, suggesting that the CCM/IDINP-induced hyperthermia effectively strengthened the chemotherapeutic effect against MG-63 cells. More strikingly, the combination of CCM/IDINPs and HRT decreased the viability of MG-63 cells to $12.6 \%$, indicating that the hyperthermia caused by CCM-camouflaged IDNPs effectively sensitized the cells to the therapeutic effect of X-ray irradiation. Moreover, the cellular uptake efficiency of CCM/IDINPs between MG-63 cells and RAW 264.7 macrophages was quantified, and the results are presented as the $1 / \mathrm{T}_{2}$ signal of SPIO. As shown in Fig. 2e, $\mathrm{f}$ and Fig. S4, CCM/IDINPs displayed higher selective cellular uptake efficiency between MG-63 cells and RAW264.7 cells than SPIO and IDINPs, indicating that the CCM-camouflaged IDINPs might be disguised as leukemic cells and thus avoid phagocytosis by macrophages in vivo.

Next, the mechanism of the strengthened anticancer ability caused by the combined treatment was examined via flow cytometry. As shown in Fig. 2g, exposure of MG63 cells to combined treatment with CCM/IDINPs +
HRT led to a significant increase in the Sub G1 and G2/M fraction compared with other treatments, suggesting that apoptosis and G2/M cell cycle arrest are involved in the cell death induced by the combined treatment.

Previously reported evidence has shown that the reactive oxygen species (ROS) generated upon laser and X-ray irradiation of chemotherapeutic agents cause functional impairment in biomacromolecules and subsequently induce apoptosis $^{26-28}$. In this study, the intracellular ROS generation after exposure to CCM/IDINPs and HRT was detected using a fluorescent probe, DCFH-DA (Fig. S5). Our results showed that the intracellular ROS level was synergistically triggered by the combined treatment with CCM/IDINPs and HRT. The significantly augmented ROS level was considered to subsequently hasten the process of apoptosis and cell cycle arrest in MG-63 cells.

\section{Sequential decomposition of CCM/IDINPs upon NIR laser and X-ray irradiation}

In this study, the CCM/IDINP was designed as a multicomponent system and was expected to sequentially release drugs in response to intratumoral GSH and laser and $\mathrm{X}$-ray irradiation. To understand the drug release mode of CCM/IDINPs under different circumstances, the morphology and release rate of CCM/IDINPs in different environments was monitored (Fig. 3a-c). As shown in Fig. 3b, c, the morphology of CCM/IDINPs remained integrated in a neutral (Phase I) and acidic environment (Phase II), which simulated the blood environment and acidic tumor microenvironment. Afterwards, the CCM/ IDINPs were incubated with $10 \mathrm{mM}$ GSH (Phase III) to simulate the high reducing environment of cancer cells. As visualized by TEM, the nanoparticles became partially detached from the CCM due to breakage of the disulfide linkage between DOX and SPIO induced by the high concentration of the reducing molecule GSH. Under this circumstance, the release rate of CCM/IDINPs was increased to $37.5 \%$ at $36 \mathrm{~h}$ of treatment. Subsequently, irradiation of CCM/IDINPs with an NIR laser (Phase IV) and X-rays (Phase V) caused large-scale drug detachment and shrinkage of the CCM. The release rate of CCM/ IDINPs was greatly hastened to $65.1 \%$ at $72 \mathrm{~h}$ and reached $83.6 \%$ by $120 \mathrm{~h}$. These results demonstrated that CCM/ IDINPs, as a multicomponent nanosystem, enabled sequential release of drugs in cancer cells in response to irradiation with a laser and X-rays. The release mode of CCM/IDINPs upon NIR laser and X-ray irradiation is displayed in Fig. 3a. Moreover, by staining the cytoskeleton of MG-63 cells with Alexa Fluor 488 phalloidin, we demonstrated that CCM/IDINPs gradually accumulated in the nucleus by $12 \mathrm{~h}$ (Fig. $3 \mathrm{~d}$ and Fig. S6). It is possible that the high concentration of GSH $(2-10 \mathrm{mM})$ in cancer cells assists in detaching the DOX from CCM/IDINPs and thus facilitates translocation of DOX into the nucleus. 


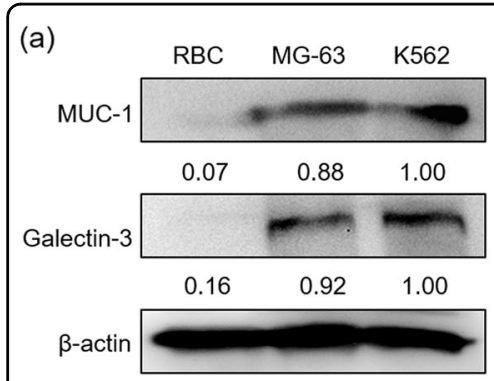

(b)

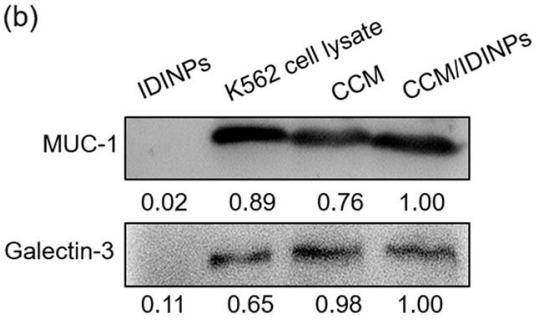

(c)

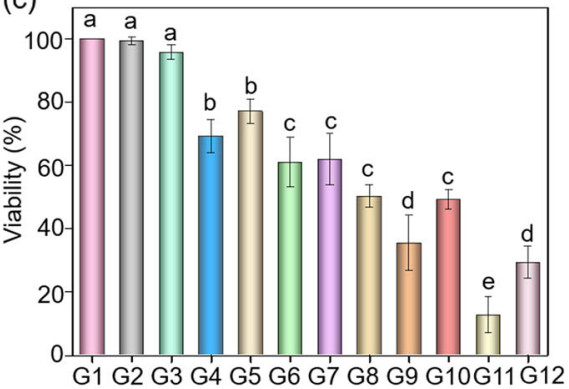

(d)

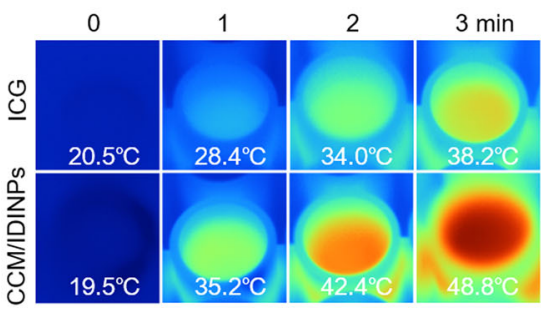

(e)

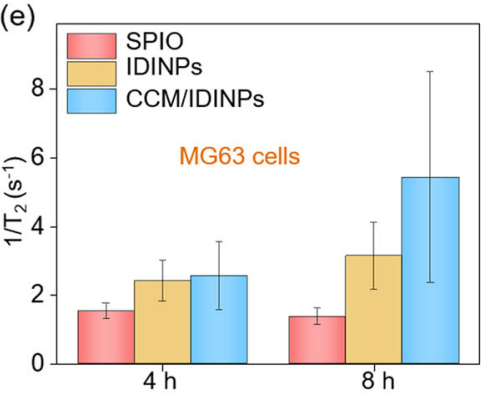

(f)

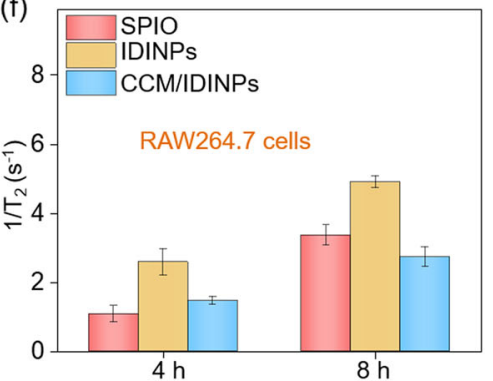

(g)
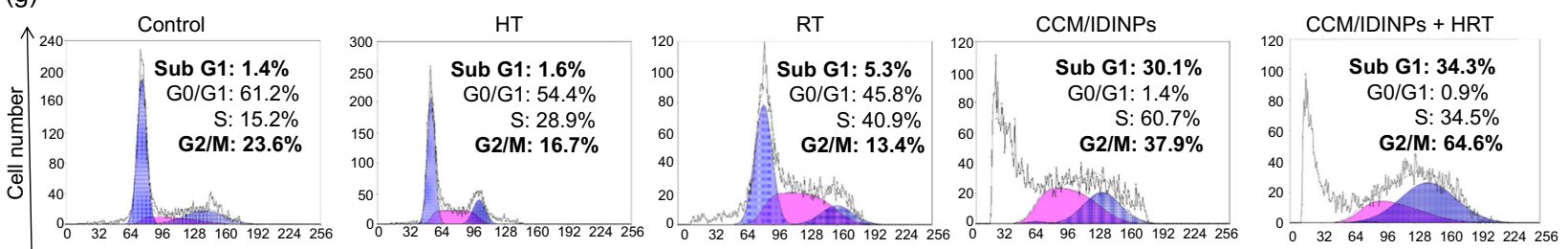

DNA content

Fig. 2 Cytotoxicity of CCM/IDINPs combined with HRT and the immune evasion capability of CCM/IDINPs. a Expression of MUC-1 and Galectin-3 on RBCs and MG-63 and K562 cells. b Expression of MUC-1 and Galectin-3 in IDINPs, K562 lysate, K562 membrane, and CCM/IDINPs. $\beta$-Actin was used as the loading control. c Cytotoxicity of CCM/IDINPs combined with HRT against MG-63 cells. The labels are presented as follows. G1: PBS; G2: NIR Laser; G3: X-ray; G4: DOX; G5: ICG + Laser; G6: SPIO@DOX; G7: IDINPs; G8: CCM/IDINPs; G9: CCM/IDINPs + HT; G10: CCM/IDINPs + RT; G11: CCM/IDINPs + HRT; G12: IDINPs + HRT. Laser power density: $1.0 \mathrm{~W} / \mathrm{cm}^{2}$; NIR irradiation time: 3 min; X-ray dosage: 2 Gy. Bars with different characters are significantly different at the $P<0.05$ level. $\mathbf{d}$ Representative images of ICG and CCM/IDINPs after irradiation with an NIR laser $\left(808 \mathrm{~nm}, 1.0 \mathrm{~W} / \mathrm{cm}^{2}\right)$ for 3 min. Cellular uptake efficacy of SPIO, IDINPs, and CCM/IDINPs in MG-63 cells (e) and RAW426.7 cells (f), as presented by the $1 / T_{2}$ value of SPIO under a 1.5 T MR scanner. g Flow cytometric analysis of MG-63 cells after treatment with CCM/IDINPs combined with HRT (Laser density: 1.0 W/cm², X-ray dosage: 2 Gy)

\section{Pharmacokinetics and biodistribution of the CCM-camouflaged nanosystem}

The size change of CCM/IDINPs in different physiological environments was evaluated in PBS, DMEM containing 10\% FBS and human plasma. As shown in Fig. 4a, the size of CCM/IDINPs was stable in human plasma for an observation period of $120 \mathrm{~h}$, indicating excellent stability of CCM/IDINPs in blood. In addition, incubation of CCM/IDINPs with red blood cells (RBCs) for $6 \mathrm{~h}$ resulted in a low degree of hemolysis compared with the DOX treatment, further demonstrating the excellent biocompatibility of CCM/IDINPs in blood circulation (Fig. S7). Next, we examined the pharmacokinetics of CCM/IDINPs and found that CCM/IDINPs, IDINPs, DOX and ICG followed two-compartment pharmacokinetics. In addition, CCM/IDINPs drastically increased the plasma concentration of DOX and ICG compared with treatment with free DOX, ICG, and IDINPs (Fig. 4b, c, Tables S1 and S2), as evidenced by the increased indices of the blood-elimination half-life (elimination phase, $t_{1 / 2} \beta$ ), maximum plasma concentration $\left(C_{\text {max }}\right)$, area under the curve $\left(\mathrm{AUC}_{0-80} \mathrm{~h}\right)$, mean retention time (MRT), and reduced clearance $(\mathrm{Cl})$ value. These results demonstrated that the nanoparticulate formulation of CCM/IDINPs improved the pharmacokinetics of free DOX, ICG, and IDINPs via favorable tumorhoming ability and the EPR effect during blood circulation.

MRI is known as a noninvasive supplementary technique to acquire spatial images of tissues and information 

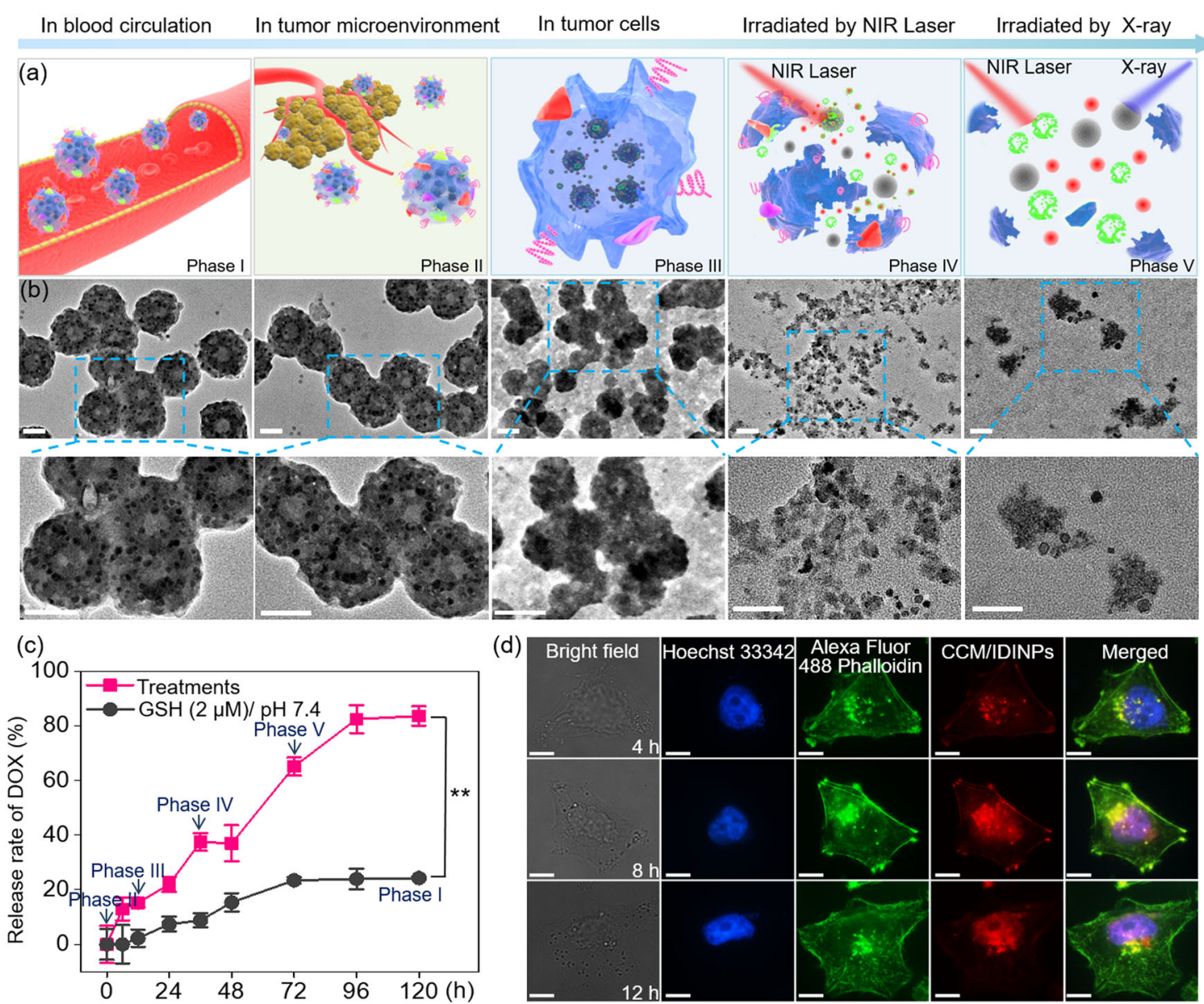

Fig. 3 Sequential decomposition of CCM/IDINPs in tumor cells upon NIR laser and X-ray irradiation. Schematic illustration (a) and TEM images

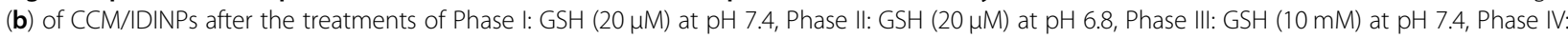
Laser + GSH (10 mM) at pH 7.4, and Phase V: Laser + X-ray + GSH $(10 \mathrm{mM})$ at pH 7.4, scale bar $=50 \mathrm{~nm}$. c Sequential release rate of DOX from CCM/ IDINPs at Phase I-V. ${ }^{* *} P<0.01$ between Phase I and Phase $V$ at $120 \mathrm{~h}$. The data are presented as the means $\pm S D ; n=3$. $\mathbf{d}$ The intracellular localization of CCM/IDINPs in MG-63 cells after $12 \mathrm{~h}$. The cytoskeleton was labeled with Alexa Fluor 488 phalloidin (green fluorescence). The nucleus was labeled with Hoechst 33342 (blue fluorescence). The intracellular localization of CCM/IDINPs was recorded by monitoring the red fluorescence of DOX; scale bar $=10 \mu \mathrm{m}$

about tumor physics, vascularity, and vascular permeability in preclinical models and clinical diagnosis ${ }^{29,30}$. In this study, the accumulation of CCM/IDINPs in the tumor region was monitored by capturing the $\mathrm{T}_{2}$-weighted signal of SPIO with a $1.5 \mathrm{~T}$ MR scanner. Our results showed stronger accumulation of CCM/IDINPs in the tumor region in $48 \mathrm{~h}$ than observed with SPIO or IDINP treatment, as shown by the stronger $\mathrm{T}_{2}$-weighted signal (Fig. 4d) and the higher percentage difference of the transverse relaxation rate $\triangle R_{2}$ " of SPIO (Fig. 4e).

To further explore the biodistribution of CCM/IDINPs in vivo, NIR fluorescence imaging (NIRFI) of ICG, IDINPs, and CCM/IDINPs was performed in nude mice by capturing the NIR signal of ICG. As shown in Fig. 4f, g, CCM-camouflaged IDINPs clearly improved the tumortargeting ability of ICG and IDINPs in vivo by increasing the accumulation of drugs in the tumor, while minimizing nonspecific accumulation in normal organs. The quantitative results showed that the tumoral amount of CCM/IDINPs was 8.0-fold and 3.4-fold higher than that of ICG and IDINPs (Fig. 4h), further demonstrating the strengthened tumor-homing ability of CCM/IDINPs in vivo.

Tumor-homing CCM/IDINPs synergize with the HRT effect and attenuate systemic toxicity in vivo

The in vivo anticancer ability of the combined treatment with CCM/IDINPs and HRT was investigated in MG-63 xenograft-bearing nude mice. Upon NIR laser irradiation for 12 days, the treatment with CCM/IDINPs generated a significantly higher temperature $\left(61.1^{\circ} \mathrm{C} \pm\right.$ 1.1) in the tumor region than that of ICG $\left(52.4{ }^{\circ} \mathrm{C} \pm 1.3\right)$ and IDINPs $\left(57.6^{\circ} \mathrm{C} \pm 1.2\right)$, due to the inherent CCMguided tumor homing ability and EPR effect in vivo 


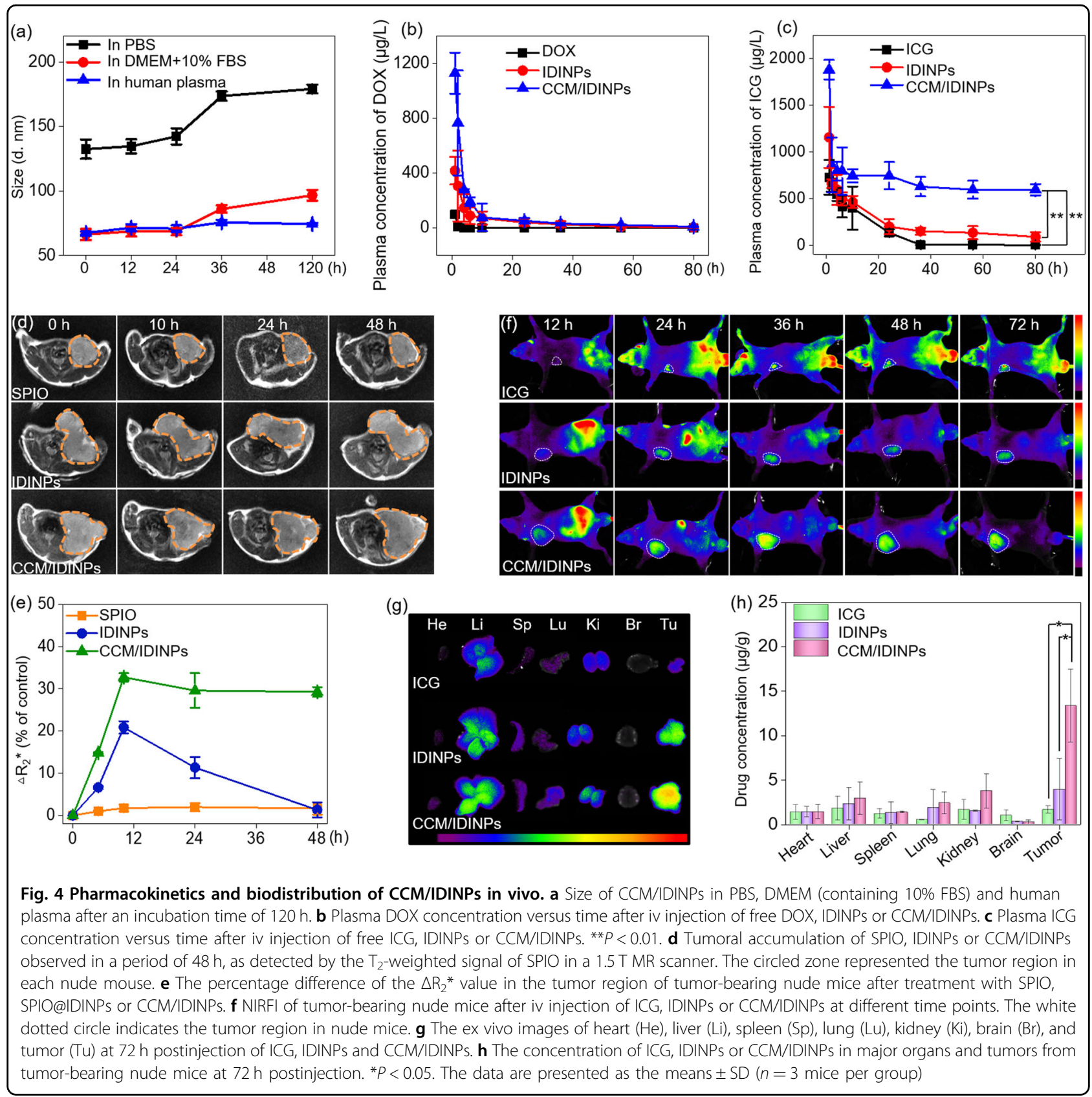

(Fig. 5a, b). In addition, we found that combined treatment with CCM/IDINPs and HT drastically enhanced the radiotherapy outcomes, as evidenced by the significantly reduced tumor volumes and weights compared with other treatments (Fig. $5 \mathrm{c}, \mathrm{d}$ ), indicating that the hyperthermia caused by CCM/IDINPs effectively antagonized the radioresistance of the tumor region. It is worth noting that treatment with IDINPs + HRT exhibited a lower anticancer effect than that of CCM/IDINPs + HRT, which verified the importance of the CCM-mediated tumor homing ability for effective cancer treatment. The tumor volume and weight results for other treatments are supplied in Figs. S8 and S9. By measuring the concentration of DOX and ICG in different organs and tumors after 19 days of treatment, we further demonstrated the effective tumoral accumulation of CCM/ IDINPs in vivo (Fig. 5e, $\mathrm{f}$ and Fig. S10).

To further investigate the activity in the tumor region after the combined treatment, MR imaging was performed. Treatment with CCM/IDINPs + HRT drastically reduced the tumor area in nude mice, which was in accordance with our previous results (Fig. 5g). Moreover, the standard apparent diffusion coefficient (ADC) and slow ADC values, which characterize the density and 

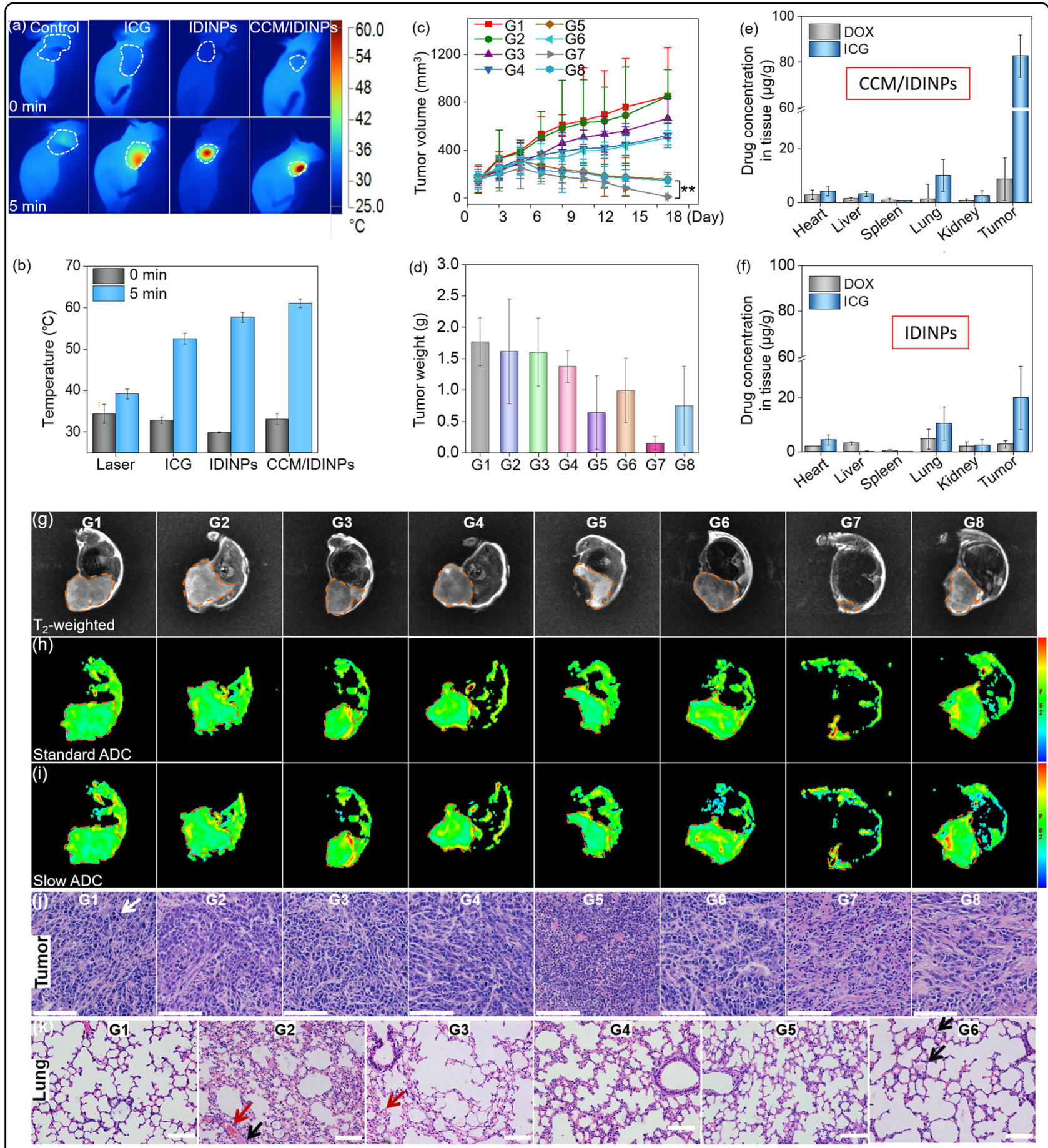

Fig. 5 Anticancer ability and systemic toxicity of CCM/IDINPs + HRT in vivo. Photothermal images (a) and temperature curves (b) of the tumor region from nude mice after irradiation with an NIR laser $\left(808 \mathrm{~nm}, 1.0 \mathrm{~W} / \mathrm{cm}^{2}\right)$ on the $12^{\text {th }}$ day of drug administration $(n=3$ mice per group). c Tumor volume of nude mice after different treatments with an observation period of 19 days; ${ }^{*} P<0.01$ between the CCM/IDINPs + HRT group and IDINPs + HRT group. $\mathbf{d}$ Tumor weights of nude mice measured on the 19th day of different treatments. The accumulative concentration of DOX and ICG in major organs and tumors was measured on the 19th day of treatment with CCM/IDINPs (e) or IDINPs (f). The data are presented as the means \pm SD ( $n=6$ mice per group). $T_{2}$-weighted MR images $(\mathbf{g})$, standard ADC $(\mathbf{h})$ and slow ADC map (i) of CCM/IDINPs + HRT-treated tumor-bearing mice on the $19^{\text {th }}$ day postinjection. The circled zone indicates the tumor region in the nude mice; $n=3$ mice per group. $\mathbf{j}$ H\&E-stained tumor region from nude mice after treatment with G1: saline; G2: HT; G3: RT; G4: CCM/IDINPs; G5: CCM/IDINPs + HT; G6: CCM/IDINPs + RT; G7: CCM/IDINPs + HRT; or G8: IDINPs + HRT. The white arrow denotes the multinuclear giant cell ( $n=3$ mice per group); scale bar $=100 \mu \mathrm{m}$. $\mathbf{k}$ Histologic lung specimens from nude mice after treatment with G1: saline; G2: DOX; G3: SPIO@DOX; G4: CCM/IDINPs; G5: CCM/IDINPs + HRT; or G6: IDINPs + HRT, stained with hematoxylin (violet) and eosin (red). The red arrows indicate hemorrhage, and the black arrow indicates the exudate in pulmonary alveoli; scale bar $=100 \mu \mathrm{m}$ 
activity of the tumor region, were enhanced by treatment with CCM/IDINPs + HRT, demonstrating reduced density and activity of the tumors in vivo (Fig. $5 \mathrm{~h}$, $\mathrm{i}$ and Fig. S11). Next, H\&E staining was performed to verify the anticancer ability by assessing the histological status. As shown in Fig. 5j, nuclear atypia and multinuclear giant cells (as denoted by the white arrow) were present in the tumor sections from the saline group, indicating that MG63-bearing nude mice were undergoing malignant tumor progression. However, combined treatment with CCM/ IDINPs and HRT caused evident nuclear condensation and drastically decreased the cell density in the tumor sections, further demonstrating the outstanding in vivo antitumor ability of CCM/IDINPs and HRT.

Safety assessment of combined therapies is of pivotal concern for application in the biomedical field. In this study, the potential systemic toxicity caused by combined treatment with CCM/IDINPs and HRT was investigated. The results of H\&E staining showed that the CCM/IDINP treatment attenuated the pulmonary edema (exudates in pulmonary alveoli, as denoted by the black arrows) and hyperemia (RBCs in pulmonary alveoli, as denoted by the red arrows) caused by DOX and IDINPs (Fig. 5k). In addition, we demonstrated that combined treatment with CCM/IDINPs and HRT was nontoxic to other major organs in the body, as shown by the histological results and body weight measurements (Figs. S12 and S13). Moreover, we demonstrated that the IDINPs + laser and $\mathrm{SPIO}+\mathrm{X}$-ray treatments were nontoxic to the healthy organs (Figs. S14 and S15). Blood biochemical analysis was conducted on the 19th day of the different treatments to investigate the function of organs in vivo (Fig. S16). As expected, the combination of CCM/IDINPs + HRT effectively attenuated the lung, liver, heart, brain, and blood pressure impairments caused by tumor progression, as shown by the indices, including GLB, UA, LDH, AST, ALT, A-G, CHOL, LDL-C, CREA, and CK, indicating the safety of CCM/IDINPs + HRT for clinical translation.

\section{Combined therapy antagonizes tumor hypoxia and promotes M1 macrophage polarization}

It is known that tumor hypoxia and TAMs residing at the site of the hypoxic region of tumors promote cancer chemo-resistance and hasten cancer cell proliferation ${ }^{31}$. Fortunately, the accumulation of hyperthermia in the tumor region has been reported to alleviate tumor hypoxia by improving tumor oxygenation status ${ }^{32,33}$ and is expected to reprogram the polarization of TAMs towards the anti-tumor M1 phenotype. To investigate whether treatment with CCM/IDINPs + HRT affects the polarization of TAMs, immunohistochemistry and flow cytometric analysis were performed. Our results showed that hypoxia-inducible factor 1-alpha (HIF1- $\alpha$ ), a critical marker of hypoxic tumor cells ${ }^{34}$, was highly expressed in the tumor region in the nontreatment group, indicating a highly hypoxic environment at the tumor sites (Fig. 6a). However, combined treatment with CCM/IDINPs + HRT drastically eradicated the expression of HIF1- $\alpha$ in the tumor region, demonstrating that the hyperthermia caused by CCM/IDINPs significantly improved oxygenation at the tumor sites and thus relieved tumor hypoxia. In addition, combined treatment with CCM/IDINPs + HRT was demonstrated to drastically increase the expression of Hsp 70, which plays an important role in tumor antigen presentation and triggering the immune response $^{35}$. Subsequently, the Hsp 70 overexpression augments the expression of TNF- $\alpha$, a crucial cytokine for initiating M1 macrophages ${ }^{36}$. The histogram and quantified results of flow cytometry shown in Fig. 6b, c indicate that combined treatment with CCM/IDINPs + HRT significantly enhanced the ratio of iNOS (marker of M1 macrophages) while evidently reducing the ratio of $\mathrm{CD} 206$ (marker of M2 macrophages) in F4/80-labeled TAMs. The enhanced M1/M2 ratio further demonstrated reprogramming of TAMs to the M1 phenotype caused by CCM/IDINPs + HRT (Fig. 6d). The mechanism by which TAM polarization was reprogrammed by CCM/IDINPs + HRT is described in Fig. 6e. These results indicated that combined treatment with CCM/IDINPs and HRT effciently relieved the tumor hypoxia and reprogramed the polarization of TAMs to an M1 phenotype, which served as a potent adjuvant to increase the specificity of the cancer treatment in vivo.

\section{Conclusion}

Rational design of smart nanosystems with dual-modal imaging properties is a critical milestone for realizing precise imaging-guided cancer theranostics. Herein, we have developed a multifunctional CCM/IDINP system with efficient tumor-homing targeting ability and photothermal and radiosensitive effects to achieve enhanced anticancer activity in vivo. In this nanosystem, the CCM coating on IDINPs provoked adhesion of CCM/IDINPs to tumor cells and accumulation at the tumor site, while alleviating nonspecific accumulation of drugs in normal tissues. Furthermore, switch-on NIR laser and X-ray irradiation provoked the release of DOX from CCM/ IDINPs in GSH-enriched cancer cells. The loading of SPIO and ICG in CCM/IDINPs facilitated precise monitoring of CCM/IDINPs in the tumor region via MRI and NIRFI. The irradiation of CCM/IDINPs with an NIR laser caused a high level of hyperthermia, which effectively antagonized tumor hypoxia and inhibited radioresistance in the tumor region by promoting polarization of the protumoral M2 TAMs towards anti-tumoral M1 macrophages. Our results confirm the feasibility of utilizing autologous cancer cells from patients for developing personalized and multimodal cancer theranostics. 


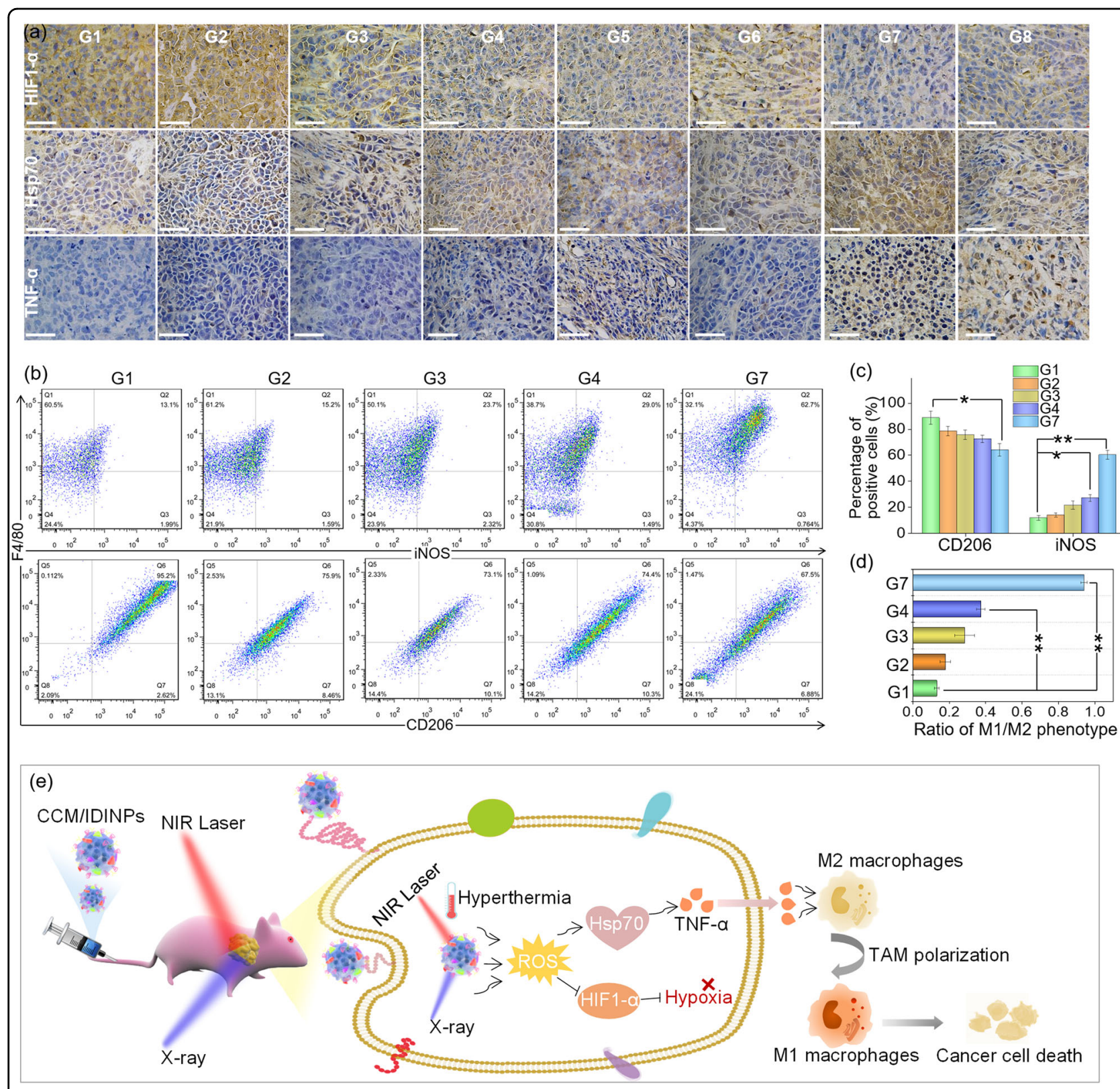

Fig. 6 Mechanism of tumor growth inhibition caused by CCM/IDINPs combined with HRT. a Immunohistochemical analysis of the expression of HIF1-a, Hsp 70 and TNF-a in tumor region slices after different treatments. Scale bar $=50 \mu \mathrm{m}$. b Flow cytometric analysis of the macrophage phenotypes in tumors after different treatments. $\mathbf{c}$ The percentage of CD206-labeled M2 macrophages and iNOS-labeled M1 macrophages. ${ }^{*} P<0.05$; ${ }^{*} P<0.01$. $\mathbf{d}$ The ratio of M1/M2 phenotype macrophages; ${ }^{* *} P<0.01$. The data are presented as the means \pm SD $(n=2$ mice per group). The labels are represented as follows. G1: saline; G2: HT; G3: RT; G4: CCM/IDINPs; G5: CCM/IDINPs + HT; G6: CCM/IDINPs + RT; G7: CCM/IDINPs + HRT; G8: IDINPs + HRT. e Overview of the mechanism of TAM polarization reprogramming by CCM/IDINPs + HRT

\section{Acknowledgements}

This work was supported by Natural Science Foundation of China (21877049 and 21701053), National High-level personnel of special support program (W02070191), Yang Fan Innovative and Entrepreneurial Research Team Project (201312 H05), and Fundamental Research Funds for the Central Universities and China Postdoctoral Science Foundation (2016M602599).

Conflict of interest

The authors declare no conflict of interest.

\section{Publisher's note:}

Springer Nature remains neutral with regard to jurisdictional claims in published maps and institutional affiliations.

Supplementary information is available for this paper at https://doi.org/ 10.1038/s41427-018-0091-9.

Received: 11 June 2018 Revised: 11 June 2018 Accepted: 19 August 2018. Published online: 12 October 2018 


\section{References}

1. Gao, M., Yu, F., Lv, C., Choo, J. \& Chen, L. Fluorescent chemical probes for accurate tumor diagnosis and targeting therapy. Chem. Soc. Rev. 46, 2237-2271 (2017)

2. Heinzmann, K., Carter, L. M., Lewis, J. S. \& Aboagye, E. O. Multiplexed imaging for diagnosis and therapy. Nat. Biomed. Eng. 1, 697-713 (2017).

3. Bruns, O. T. et al. Next-generation in vivo optical imaging with short-wave infrared quantum dots. Nat. Biomed. Eng. 1, 1-20 (2017).

4. Fan, W., Yung, B., Huang, P. \& Chen, X. Nanotechnology for multimodal synergistic cancer therapy. Chem. Rev. 117, 13566-13638 (2017).

5. Datta, N. R. et al. Local hyperthermia combined with radiotherapy and-/or chemotherapy: recent advances and promises for the future. Cancer Treat. Rev. 41, 742-753 (2015)

6. Lee, S. Y., Lee, N. R., Cho, D. H. \& Kim, J. S. Treatment outcome analysis of chemotherapy combined with modulated electro-hyperthermia compared with chemotherapy alone for recurrent cervical cancer, following irradiation. Oncol. Lett. 14, 73-78 (2017).

7. Hu, Y. et al. Chemoradiation combined with regional hyperthermia for advanced oesophageal cancer: a systematic review and meta-analysis. J. Clin. Pharm. Ther. 42, 155-164 (2017).

8. $\mathrm{Wu}$, L. et al. Hybrid polypeptide micelles loading indocyanine green for tumor imaging and photothermal effect study. Biomacromolecules 14, 3027-3033 (2013).

9. Yang, W. et al. Near infrared dye-conjugated oxidative stress amplifying polymer micelles for dual imaging and synergistic anticancer phototherapy. Biomaterials 154, 48-59 (2018).

10. Ojha, T. et al. Pharmacological and physical vessel modulation strategies to improve EPR-mediated drug targeting to tumors. Adv. Drug Deliv. Rev. 119, 44-60 (2017).

11. He, L. Z. et al. A highly selective dual-therapeutic nanosystem for simultaneous anticancer and antiangiogenesis therapy. J. Mater. Chem. B 5, 8228-8237 (2017).

12. Jin, Q. T. et al. Ultra-small iron-gallic acid coordination polymer nanoparticles for chelator-free labeling of Cu-64 and multimodal imaging-guided photothermal therapy. Nanoscale 9, 12609-12617 (2017).

13. Long, M. J. C., Poganik, J. R. \& Aye, Y. On-demand targeting: investigating biology with proximity-directed chemistry. J. Am. Chem. Soc. 138, 3610-3622 (2016).

14. Hartshorn, C. M. et al. Nanotechnology strategies to advance outcomes in clinical cancer care. ACS Nano 12, 24-43 (2018).

15. Zanganeh, S. et al. Iron oxide nanoparticles inhibit tumour growth by inducing pro-inflammatory macrophage polarization in tumour tissues. Nat. Nanotechnol. 11, 986-994 (2016).

16. Zhang, P. F., Liu, G. \& Chen, X. Y. Nanobiotechnology: cell membrane-based delivery systems. Nano Today 13, 7-9 (2017).

17. Fang, R. H. et al. Cancer cell membrane-coated nanoparticles for anticancer vaccination and drug delivery. Nano Lett. 14, 2181-2188 (2014).

18. Zhao, P. et al. Improving drug accumulation and photothermal efficacy in tumor depending on size of ICG loaded lipid-polymer nanoparticles. Biomaterials 35, 6037-6046 (2014).
19. Gao, M. et al. Erythrocyte-membrane-enveloped perfluorocarbon as nanoscale artificial red blood cells to relieve tumor hypoxia and enhance cancer radiotherapy. Adv. Mater. 29, 1701429 (2017)

20. Chen, Z. et al. Cancer cell membrane-biomimetic nanoparticles for homologous-targeting dual-modal imaging and photothermal therapy. ACS Nano 10, 10049-10057 (2016).

21. Zhu, J. Y. et al. Preferential cancer cell self-recognition and tumor self-targeting by coating nanoparticles with homotypic cancer cell membranes. Nano Lett. 16, 5895-5901 (2016)

22. Li, S. Y. et al. Cancer cell membrane camouflaged cascade bioreactor for cancer targeted starvation and photodynamic therapy. ACS Nano 11, 7006-7018 (2017)

23. Huang, Y. Y. et al. Selective cellular uptake and induction of apoptosis of cancer-targeted selenium nanoparticles. Biomaterials 34, 7106-7116 (2013).

24. Huang, Y., Luo, Y., Zheng, W. \& Chen, T. Rational design of cancer-targeted BSA protein nanoparticles as radiosensitizer to overcome cancer radioresistance. ACS Appl. Mater. Interfaces 6, 19217-19228 (2014).

25. Naumova, A. V., Modo, M., Moore, A., Murry, C. E. \& Frank, J. A. Clinical imaging in regenerative medicine. Nat. Biotechnol. 32, 804-818 (2014).

26. Chang, K. W. et al. Enhanced phototherapy by nanoparticle-enzyme via generation and photolysis of hydrogen peroxide. Nano Lett. 17, 4323-4329 (2017).

27. Nathan, C. \& Cunningham-Bussel, A. Beyond oxidative stress: an immunologist's guide to reactive oxygen species. Nat. Rev. Immunol. 13, 349-361 (2013).

28. Pisoschi, A. M. \& Pop, A. The role of antioxidants in the chemistry of oxidative stress: a review. Eur. J. Med. Chem. 97, 55-74 (2015).

29. Zhang, F., Zhu, L., Huang, X., Niu, G. \& Chen, X. Differentiation of reactive and tumor metastatic lymph nodes with diffusion-weighted and SPIO-enhanced MRI. Mol. Imaging Biol. 15, 40-47 (2013).

30. Liu, G. et al. A dextran-based probe for the targeted magnetic resonance imaging of tumours expressing prostate-specific membrane antigen. Nat Biomed. Eng. 1, 977-982 (2017).

31. Song, M. L., Liu, T., Shi, C. R., Zhang, X. Z. \& Chen, X. Y. Bioconjugated manganese dioxide nanoparticles enhance chemotherapy response by priming tumor-associated tL.A macrophages toward M1-like phenotype and 11 attenuating tumor hypoxia. ACS Nano 10,633-647 (2016).

32. Yang, Y. et al. Core-shell and co-doped nanoscale metal-organic particles (NMOPs) obtained via post-synthesis cation exchange for multimodal imaging and synergistic thermo-radiotherapy. NPG Asia Mater. 9, 1-11 (2017).

33. Yang, G. B. et al. Hollow $\mathrm{MnO}_{2}$ as a tumor-microenvironment-responsive biodegradable nano-platform for combination therapy favoring antitumor immune responses. Nat. Commun. 8, 902 (2017).

34. Kang, R. et al. RAGE is essential for oncogenic KRAS-mediated hypoxic signaling in pancreatic cancer. Cell Death Dis. 5, 1-10 (2014).

35. Robbins, P. D. \& Morelli, A. E. Regulation of immune responses by extracellular vesicles. Nat. Rev. Immunol. 14, 195-208 (2014).

36. Genard, G., Lucas, S. \& Michiels, C. Reprogramming of tumor-associated macrophages with anticancer therapies: radiotherapy versus chemo- and immunotherapies. Front. Immunol. 8, 1-19 (2017). 